\title{
An Appraisal of the Influence of the Metabotropic Glutamate 5 (mGlu5) Receptor on Sociability and Anxiety
}

\author{
Arnau Ramos-Prats, Julia Kölldorfer, Elena Paolo, Maximilian Zeidler, Gabriele Schmid \\ and Francesco Ferraguti*
}

Department of Pharmacology, Medical University of Innsbruck, Innsbruck, Austria

Amongst the many neurotransmitter systems causally linked to the expression of social behavior, glutamate appears to play a pivotal role. In particular, metabotropic glutamate 5 (mGlu5) receptors have received much attention as its altered function has been reported in several mouse models of autism spectrum disorders and mental retardation. Inhibition of the activity of mGlu5 receptors by means of genetic or pharmacological manipulations improved social deficits in some of these animal models. However, in normal wild-type (WT) mice, pharmacological blockade of mGlu5 receptors yielded inconsistent results. The aim of our study was to investigate the actual contribution of decreased or absent mGlu5 receptor function in sociability and anxiety-like behavior as well as to explore the impact of mGlu5 receptor ablation on the pattern of brain activation upon social exposure. Here we show that Grm5-/- mice display higher social preference indexes compared to age-matched WT mice in the three-chambered social task. However, this effect was accompanied by a decreased exploratory activity during the test and increased anxiety-like behavior. Contrary to

OPEN ACCESS

Edited by:

Enza Palazzo,

Second University of Naples, Italy

Reviewed by:

Andrzej Pilc,

Polish Academy of Sciences, Poland

Camilla Bellone,

Université de Genève, Switzerland

*Correspondence:

Francesco Ferraguti

francesco.ferraguti@i-med.ac.at

Received: 07 August 2018 Accepted: 24 January 2019

Published: 19 February 2019

Citation:

Ramos-Prats A, Kölldorfer J, Paolo E, Zeidler M, Schmid G and Ferraguti F (2019) An Appraisal of the Influence of the Metabotropic Glutamate 5 (mG/u5) Receptor on Sociability and Anxiety.

Front. Mol. Neurosci. 12:30. doi: 10.3389/fnmol.2019.00030 mGlu5 receptor ablation, the mGlu5 receptor negative allosteric modulator 3-((2-methyl1,4-thiazolyl)ethynyl)pyridine (MTEP) induced anxiolytic effects without affecting social preference in WT mice. By mapping c-Fos expression in 21 different brain regions known to be involved in social interaction, we detected a specific activation of the prefrontal cortex and dorsolateral septum in Grm5-/- mice following social interaction. C-Fos expression correlation-based network and graph theoretical analyses further suggested dysfunctional connectivity and disruption of the functional brain network generated during social interaction in Grm5-/- mice. The lack of mGlu5 receptors resulted in profound rearrangements of the functional impact of prefrontal and hippocampal regions in the social interaction network. In conclusion, this work reveals a complex contribution of mGlu5 receptors in sociability and anxiety and points to the importance of these receptors in regulating brain functional connectivity during social interaction.

Keywords: social behavior, glutamate receptors, anxiety, brain networks, MTEP

\section{INTRODUCTION}

The group I metabotropic glutamate 5 (mGlu5) receptor couples to $\mathrm{G} \alpha \mathrm{q} / 11$ proteins to activate a number of intracellular signaling cascades (Hermans and Challiss, 2001; Nicoletti et al., 2011) and regulates synaptic activity and plasticity (Manahan-Vaughan and Braunewell, 2005; Homayoun and Moghaddam, 2010). This receptor is abundantly expressed in telencephalic brain areas 
(Ferraguti and Shigemoto, 2006) involved in learning and memory, emotions and in the control of movement and it was found to contribute to a variety of behaviors ranging from cognition to sensory-motor gating and novelty-induced locomotion (Lu et al., 1997; Kinney et al., 2003; Brody et al., 2004; Jew et al., 2013).

A large body of evidence has implicated an altered mGlu5 receptor signaling or expression in the pathology of several neuropsychiatric disorders, including autism, schizophrenia, and anxiety (Nicoletti et al., 2011; Bhakar et al., 2012; D'Antoni et al., 2014; Matosin et al., 2017; Ferraguti, 2018). In particular, enhanced mGlu5 receptor activity has been suggested as one of the underlying mechanisms contributing to several symptoms of fragile X (FX) syndrome, the most common inherited form of intellectual disability (Bhakar et al., 2012). FX-like phenotypes, such as impaired sociability, could be corrected in Fmr1 knockout mice by reducing the activity of mGlu5 receptors using both genetic and chronic pharmacological treatments (Dölen et al., 2007; Thomas et al., 2011; Bhakar et al., 2012; Gantois et al., 2013). Likewise, altered mGlu5 receptor function was reported in other mouse models of autism spectrum disorders (ASD) and mental retardation (Burket et al., 2011; Chung et al., 2015; Tian et al., 2015; Tao et al., 2016; Vicidomini et al., 2017). The "mGlu5 receptor theory of FX" was recently tested in several phase II, placebo controlled, clinical trials, which, however, did not achieve significant efficacy in the primary end point of improvement on behavioral symptoms (Berry-Kravis et al., 2016; Youssef et al., 2018). Despite the big disappointment and conjectures on the validity of the theory, a number of caveats characterizing these trials may explain why they have failed. First and foremost, the scales of treatment response used in these studies could be biased by caregiver or family involvement in ratings, thus lacking truly quantitative and objective measures of behavioral and cognitive performance. These assessment scales are also known to be subject to a strong placebo effect. Moreover, the study duration and the doses of mGlu5 receptor antagonists utilized might have been inadequate based on preclinical animal data. These results, besides highlighting the difficulty of translating findings from animal models to humans, also call for a new appraisal of the role of mGlu5 receptors in distinct behaviors.

For instance, the influence of mGlu5 receptor antagonism on different aspects of social behavior in wild-type (WT) rodents has been poorly investigated and the current literature contains a number of inconsistent results. The negative allosteric modulator (NAM) 3-((2-methyl-1,4-thiazolyl)ethynyl)pyridine (MTEP) was shown to induce social isolation in rats (Koros et al., 2007), whereas both AFQ056/Mavoglurant and 2methyl-6-(phenylethynyl)pyridine (MPEP), when administered systemically, elicited no substantial effects on sociability in WT mice (Gantois et al., 2013; Chung et al., 2015). It remains, therefore, unclear whether the complex effects of mGlu5 receptor antagonism in social behavior are due to the limited specificity of systemic pharmacological approaches, species differences in receptor occupancy or pharmacokinetic differences of distinct mGlu5 receptor antagonists (Anderson et al., 2003).

Another complex aspect in the study of mGlu5 receptor in social behavior is its controversial interaction with anxiety.
Besides ASD, social function is severely affected in patients with anxiety disorders. The high co-morbidity between social deficits and anxiety can be partially explained by the shared circuitry underlying both anxiety-related and social behavior (for review see: Allsop et al., 2014). Several studies have reported anxiolytic properties of different mGlu5 receptor antagonists, both in animal research and in humans (for review see: Ferraguti, 2018). Nonetheless, to which extent mGlu5 receptor modulation simultaneously regulate anxiety and social behavior in healthy and pathological conditions has not been investigated in depth. Only one study has addressed the role of mGlu5 receptors in the acquisition, expression and extinction of social anxiety in rodent models (Slattery et al., 2017). Overall, the role of mGlu5 receptors in social behavior remains unclear. In the present study, we sought to reassess the effect of mGlu5 receptor ablation or mGlu5 receptor negative allosteric modulation in social preference and anxiety-like behavior, using the classical three-chambered social task (Moy et al., 2004; Nadler et al., 2004) and the light-dark test, respectively. Furthermore, we have investigated the influence of mGlu5 receptors on brain activity patterns upon social and non-social investigation using c-Fos expression as a marker of neuronal activation. Based on these data, we further explored interregional functional connectivity using network analysis to understand at the anatomical level where mGlu5 receptors may regulate functional brain connectivity during social exploration.

\section{MATERIALS AND METHODS}

All procedures involving animals were approved by the Austrian Animal Experimentation Ethics Board and were performed in compliance with the European Convention for the Protection of Vertebrate Animals used for Experimental and Other Scientific Purposes (ETS no. 123). Every effort was taken to minimize the number of animals used. mGlu5 receptor knock-out (Grm5-/-) mice (Lu et al., 1997), were backcrossed to C57BL/6J from Charles River Laboratories (Sulzfeld, Germany) for at least 10 generations. Because Grm5-/- female mice have deficits in maternal care, breeding was carried out using homozygous male Grm5-1- and heterozygous females. Animals were weaned at 4 weeks of age and grouped housed in a climate-controlled facility on a $12 \mathrm{~h} / 12 \mathrm{~h}$ light/dark cycle with lights on at 07:00 AM, with water and food ad libitum. Genotyping was performed from ear punches and determined by PCR. Since the breeding strategy or postnatal mothering of Grm5-/+ mice were shown not to influence the offspring behavioral phenotype (Brody and Geyer, 2004), age-matched wild type (WT) C57BL/6J mice (purchased from Charles River Laboratories, Sulzfeld) were selected, instead of WT littermates, as control animals for Grm5-/- mice as well as for pharmacological experiments. C57BL/6J mice were allowed to acclimatize for at least 2 weeks before any experimental procedure. Only adult (11-18 weeks old) male mice were used. All experiments were performed during the light cycle. Prior to all experiments, animals were acclimatized to the testing room for at least 2 days.

For pharmacological experiments, mice were injected i.p. with MTEP (Hello Bio; Bristol, United Kingdom; 10 mg/kg diluted 
in $4 \%$ dimethylsulfoxide in saline) or vehicle. Mice were tested 5 min or 1-h post i.p. injection of MTEP. Dose and time post i.p. injection were chosen based on previous studies on behavioral activity and receptor occupancy of MTEP (Anderson et al., 2003; Busse et al., 2004; Nagel et al., 2015).

\section{Three-Chambered Social Task}

Social behavior was assessed by means of a modified threechambered social task apparatus (Moy et al., 2004; Nadler et al., 2004). The chamber was an opaque glass rectangular box $(75 \mathrm{~cm}$ long $\times 30 \mathrm{~cm}$ wide $\times 35 \mathrm{~cm}$ tall $)$ divided into three equal compartments, connected through small rectangular doors $(7 \mathrm{~cm} \times 7 \mathrm{~cm})$ allowing free access into each chamber. Different illumination conditions were used for pharmacological (infrared light; Lux < 5) and non-pharmacological experiments $($ Lux $<30)$. The procedure involved two phases: habituation and sociability. The test mouse was first placed in the middle chamber and allowed to explore all three chambers for $10 \mathrm{~min}$. After this habituation period, a novel unfamiliar mouse (sex, strain and age-matched) was placed into a mesh cylinder $(15 \mathrm{~cm}$ tall, $7 \mathrm{~cm}$ diameter) in the least explored side chamber, whereas an identical empty mesh cylinder was placed in the opposite chamber. The mesh cylinder allowed for air exchange, visual, olfactory and auditory interaction, but prevented fighting. The test mouse was then allowed to explore the chambers for $10 \mathrm{~min}$ (sociability). Measurements during the test phase included: distance traveled, latency to explore side chambers at the beginning of the test, time spent in each chamber and in close proximity to the mesh cylinders $(<5 \mathrm{~cm})$. Tracking and scoring was performed using Ethovision XT 10 software (Noldus; RRID:SCR_000441). The social preference index for conspecific chamber time was calculated as follows:

$$
\frac{(T c-T o)}{(T c+T o)} \times 100
$$

Tc = Time spent in conspecific chamber

To $=$ Time spent in object chamber

whereas the social preference index for time in close interaction was calculated as follows:

$$
\frac{(\operatorname{Tnc}-T n o)}{(\operatorname{Tnc}+\operatorname{Tno})} \times 100
$$

Tnc $=$ Time spent in close proximity to the conspecific

Tno $=$ Time spent in close proximity to the object

\section{Object Interaction Task}

Object interaction was assessed in an identical apparatus as described above. Under infrared light conditions, mice were first allowed to explore the three chambers of the empty apparatus for $10 \mathrm{~min}$. After this habituation, an empty mesh cylinder was placed in the most preferred side chamber and mice were allowed to freely explore the apparatus for $30 \mathrm{~min}$. Tracking and scoring was performed using Ethovision XT 10 software (Noldus; RRID:SCR_000441). Time spent in the object chamber was scored and expressed as the percentage of total test time.

\section{Light-Dark Test}

Anxiety-like behavior was tested using the light-dark test. The apparatus (TSE Systems, Bad Homburg, Germany) consisted of a dark (<10 Lux) "safe" compartment and an illuminated aversive compartment (400 Lux). The compartments were connected by a small opening $(7 \mathrm{~cm} \times 7 \mathrm{~cm}$ wide) located in the center of the partition at floor level. Animals were individually placed in the apparatus facing the opening to the dark compartment and allowed to freely explore the apparatus for $10 \mathrm{~min}$. Time spent in the light compartment was measured using Ethovision (Noldus). In pharmacological experiments, mice tested $5 \mathrm{~min}$ after injection in the three-chambered social task were re-used after $48 \mathrm{~h}$ in the light-dark test using a counterbalanced design. Conversely, independent mouse cohorts were used for the $1 \mathrm{~h}$ post-injection experiments.

\section{Forced Social and Object Interaction for c-Fos Induction}

Adult male WT and Grm5-/- mice were single-housed for $72 \mathrm{~h}$ prior to the test. Mice from each genotype were divided into three groups ( $n=7$ /group): A group exposed to an empty mesh cylinder (object) in the home cage for $10 \mathrm{~min}$, a second group exposed in the home cage to a mesh cylinder enclosing an unfamiliar sex and age-matched mouse (conspecific) for $10 \mathrm{~min}$ and a control group maintained undisturbed in the home cage (HC). After the test, the cylinder was removed and the mice left in their home cages undisturbed. Mice were then perfused $2 \mathrm{~h}$ after the end of the experimental manipulation. Tracking and scoring of the time spent in close proximity $(<3 \mathrm{~cm})$ with the object or conspecific was performed using the Ethovision XT 10 software (Noldus). Measurement of exploration time of the novel object or novel conspecific was obtained from an independent batch of mice from those used for c-Fos quantification.

\section{Immunocytochemistry}

Mice were deeply anesthetized by i.p. injection of Thiopental $(150 \mathrm{mg} / \mathrm{kg})$ and were perfused with a fixative made of $4 \% \mathrm{w} / \mathrm{v}$ paraformaldehyde and $15 \% \mathrm{v} / \mathrm{v}$ of a saturated solution of picric

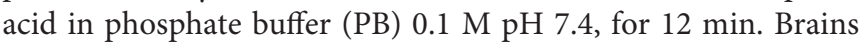
were removed from the skull, washed in $0.1 \mathrm{M} \mathrm{PB}$ and sliced coronally in $50 \mu \mathrm{m}$-thick sections on a vibratome (VT1000S, Leica Microsystems, Vienna, Austria).

Immunocytochemistry was performed as previously described (Sreepathi and Ferraguti, 2012). Briefly, free-floating sections were first washed with Tris-buffered saline (TBS; $0.9 \% \mathrm{NaCl}$, $\mathrm{pH} 7.4$ ) and then incubated in $20 \%$ normal horse serum in TBS and $0.3 \%$ Triton X100 (TX) for $1 \mathrm{~h}$ at room temperature (RT, $\left.21-23^{\circ} \mathrm{C}\right)$. After blocking, sections were incubated with a polyclonal goat primary antibody against c-Fos (1:300; Santa Cruz Biotechnology, Santa Cruz, CA, United States, catalog \#sc52 , lot \#F1112) for $\sim 72 \mathrm{~h}$ at $6^{\circ} \mathrm{C}$. After three washes in TBS, the biotinylated secondary antibody (horse anti-goat IgG 1:500, Vector Laboratories, Burlingame, CA, United States, catalog \#BA-9500,) was applied overnight at $6^{\circ} \mathrm{C}$ at a dilution of 1:500 in a buffer with the same composition as for the primary antibody. The sections were then washed and incubated in Vectastain 
elite ABC complex (diluted 1:100; Vector Laboratories) in TBS at RT for $1 \mathrm{~h}$. Subsequently, the sections were washed in TB several times, pre-incubated with $3,3^{\prime}$-diaminobenzidine (DAB; $0.5 \mathrm{mg} / \mathrm{ml}$ ) for $10 \mathrm{~min}$ and then $\mathrm{H}_{2} \mathrm{O}_{2}$ was added to the solution at a final dilution of $0.003 \%$ for $2-5 \mathrm{~min}$. Sections were then washed with TBS, mounted in gelatin onto glass slides, air-dried, and then treated with graded ethanol (50, 70, 90, 95, and 100\%) and butyl acetate. Finally, slides were coverslipped with Eukitt (Agar Scientific Ltd., Stansted, United Kingdom).

The following brain structures, relevant for social behavior (Kim et al., 2015), were selected for c-Fos quantification and identified based on the mouse brain atlas of Franklin and Paxinos (2007): medial orbital cortex (MO; bregma between $+2,8$ and $+2,22 \mathrm{~mm}$ ), prelimbic cortex (PrL; bregma between $+2,34$ and $+1,54 \mathrm{~mm}$ ), infralimbic cortex (IL; bregma between $+1,94$ and $+1,54 \mathrm{~mm}$ ), accumbens nucleus shell (AcbSh; bregma between $+1,42$ and $+1,18 \mathrm{~mm}$ ), accumbens nucleus core (AcbC; bregma between $+1,42$ and $+1,18 \mathrm{~mm}$ ), lateral septal nucleus intermedial part (LSI; bregma between $+0,62$ and $+0,14 \mathrm{~mm}$ ), lateral septal nucleus dorsal part (LSD; bregma between $+0,62$ and $+0,14 \mathrm{~mm}$ ), piriform cortex (Pir; bregma between $+0,98$ and $+0,50 \mathrm{~mm}$ ), medial septal nucleus (MS; bregma between $+0,98$ and $+0,50 \mathrm{~mm}$ ), medial preoptic nucleus medial part (MPOM; bregma between $+0,0,02$ and $-0,22 \mathrm{~mm}$ ), paraventricular thalamic nucleus (PV; bregma between $-0,22$ and $-0,58 \mathrm{~mm}$ ), paratenial thalamic nucleus (PT; bregma between $-0,22$ and $-0,58 \mathrm{~mm}$ ), reuniens thalamic nucleus (RE; bregma between $-0,46$ and $-0,70 \mathrm{~mm}$ ), basolateral amygdala (BLA; bregma between $-0,94$ and $-1,46 \mathrm{~mm}$ ), dorsal hippocampus (CA1, CA2, CA3, DG, bregma between $-1,58$ and $-1,94 \mathrm{~mm}$ ), lateral hypothalamic area ( $\mathrm{LH}$; bregma between $-2,18$ and $-2,46 \mathrm{~mm}$ ), posteromedial cortical amygdaloid area (PMCo; bregma between $-2,18$ and $-2,46 \mathrm{~mm}$ ) and periaqueductal gray (PAG; bregma between $-2,92$ and $-3,16 \mathrm{~mm}$ ). The number of $\mathrm{c}$-Fos positive cells/area was semi-automatically counted with the Neurolucida software (Version 11, MBF Bioscience, RRID:SCR_001775) coupled to an Olympus BX51 Microscope by an experimenter blinded to the treatment condition and genotype. Each brain area was analyzed bilaterally across at least three sections using a sampling window $(200 \mu \mathrm{m} \times 200 \mu \mathrm{m})$ placed always in the same position within the selected area.

\section{Brain Network Construction and Graph Theoretical Analysis}

Network analyses were performed as previously described (Tanimizu et al., 2017a). Briefly, Pearson $r$-values from interregional c-Fos expression data from home cage, objectexposed and conspecific-exposed groups from both genotypes were obtained and used to generate correlation matrices. In order to compare average correlations between groups/genotypes, $r$-values were transformed to Fischer $Z$-values, statistics were calculated, and values were retransformed to $r$ values for graph representation. To characterize the generated social and object interaction networks in both genotypes, positive $(r>0.60)$ interregional c-Fos correlations with a significance level of $p<0.05$ were used to generate unweighted network graphs.
Community clustering to generate weighted network graphs was performed based on modularity optimization, according to Newman and Girvan (2004). Finally, participation coefficient and within-community $z$ scores were calculated as defined in Guimerà and Amaral, 2005 and plotted as described by Tanimizu et al. (2017a) in order to visualize the main hubs in the generated networks. Interregional correlation matrices were obtained with Prism 7 software (GraphPad Software Inc., RRID:SCR_002798). Network construction and visualization were performed in $\mathrm{R}$ (version 3.3.3) using the igraph (version 1.1.2; Csardi and Nepusz, 2006) and brainGraph (version 1.0.0) packages.

\section{Data Analysis}

Data were analyzed with the Prism 7 software (GraphPad Software Inc.) using two-tailed Student's $t$-tests or analysis of variance (one-way or two-way ANOVA, factorial or repeated measures). Whenever an ANOVA resulted significant, HolmSidak post hoc comparisons were applied to analyze the effects of group, genotype, treatment, time and chamber in the behavioral experiments. Two-way ANOVA followed by a post hoc NewmanKeuls comparison was used to analyze the effects of genotype and groups in the c-Fos mapping experiment. Two-way ANOVA followed by a post hoc Bonferroni comparison was used to analyze differences in network density. Data were considered significant when $p<0.05$.

\section{RESULTS}

\section{Effects of mGlu5 Receptor Ablation on Sociability and Anxiety-Like Behavior}

We investigated the role of mGlu5 receptors in sociability using the classical three-chambered social task apparatus, where sociability is measured as the preference for interacting with an enclosed conspecific placed in one of the side chambers as compared to a novel object (an empty cage) placed in the opposite side chamber. At first, we examined germ-line Grm5-1mice and compared them to age-matched WT C57BL/6j mice. During the sociability test, both Grm5-/- and WT control mice displayed sociability, spending significantly more time in the social chamber than in the novel object chamber [2-way ANOVA: chamber $F(2,90)=133, p<0.001$; chamber $\times$ genotype $F(2,90)=8.06, p<0.001$; novel object vs. novel mouse chamber: WT: $p<0.001$; Grm5-/-: $p<0.001$ ] (Figures 1A,B). However, Grm5-1- mice spent less time than WT mice investigating the novel object chamber $(p<0.05)$ and spent significantly more time in the middle chamber during the test $(p<0.05)$. Similarly, time in close proximity to the novel mouse was higher than for the novel object for both genotypes [2-way ANOVA: close interaction $F(1,60)=49.7 p<0.001$, and genotype $F(1,60)=6.97, p<0.05]$ (Figure 1C), whereas the overall time in close interaction with the conspecific or object did not differ between genotypes [2-way ANOVA: close interaction $\times$ genotype $F(1,60)=2.52 p=0.11]$. Grm5-/- mice showed a higher social preference index for both conspecific chamber time [ $t$ test: $t(1,30)=2.18, p<0.05$ ] (Figure 1D) and time in close interaction with the conspecific [ $t$-test: $t(1,30)=3.178, p<0.01]$ 


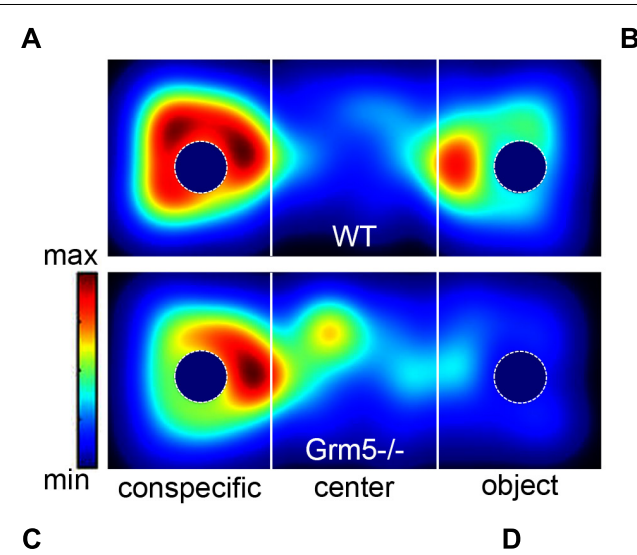

C

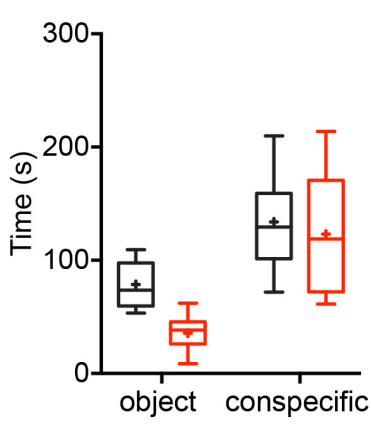

F

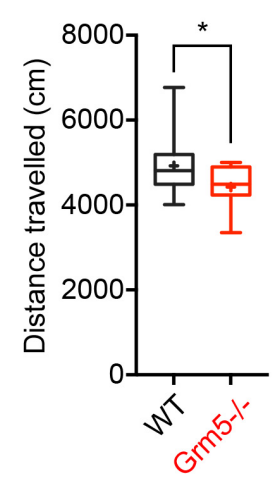

G

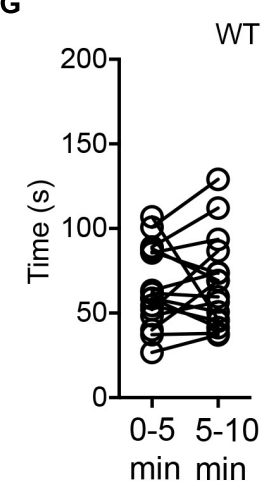

B

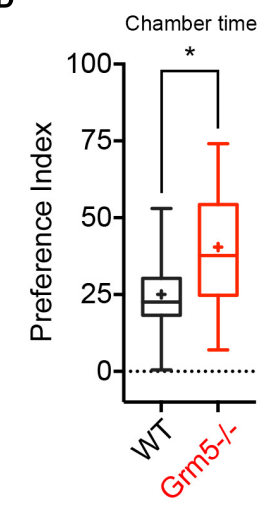

H

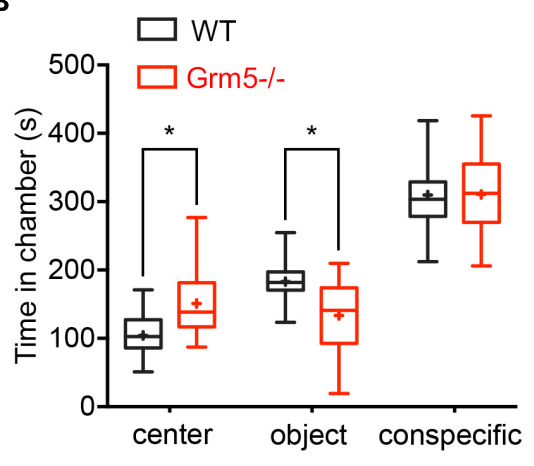

E

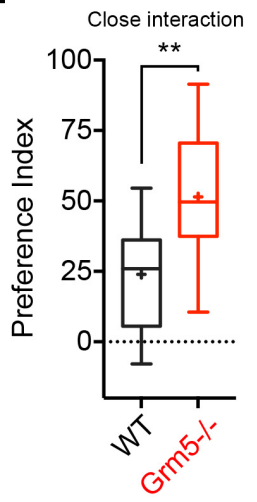

I

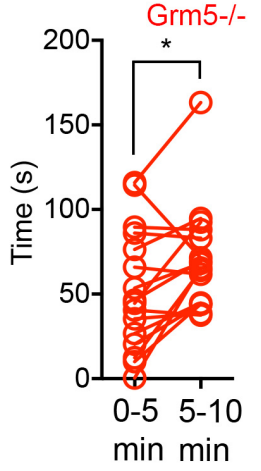

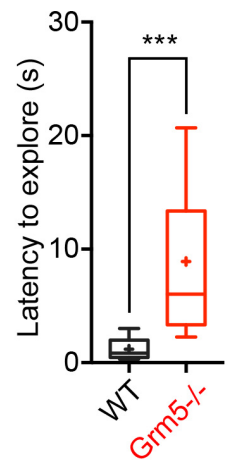

FIGURE 1 | Effects of mGlu5 receptor ablation on sociability. (A) Representative heat maps showing time spent by WT mice (upper panel) and Grm5-/- (lower panel) mice at each location of the three-chambered apparatus during the test. (B) Time spent (s) in the different compartments of the three-chambered apparatus by Grm5-/- $(n=16)$ and WT mice $(n=16)$ during the test. (C) Time spent (s) in close proximity to the social (conspecific) and non-social (object) stimulus.

(D) Preference index derived from the numerical difference between time spent in conspecific and object chamber divided by total time spent $\times 100$; and (E) index derived from the numerical difference between time spent in close proximity to conspecific and object divided by total time spent in close proximity $\times 100$.

(F) Distance traveled during the test by Grm5-/- and WT mice. (G) Time spent in close proximity to the conspecific during the first and last 5 min of the test by WT and (H) Grm5-/- mice. (I) Latency (s) to explore side chambers at the beginning of the three-chambered social task was longer for Grm5-/- mice. Boxplots represent median, upper and lower quartiles with 10th and 90 th percentile whiskers. Mean is represented with a cross. ${ }^{*} p<0.05$, ${ }^{* *} p<0.01,{ }^{* * *} p<0.001$.

(Figure 1E) when compared to control mice. During the test we observed that the total distance traveled by Grm5-/mice was significantly lower than the control animals [t-test: $t(1,30)=2.32, p<0.05$ ] (Figure 1F). Since Grm5-/- mice are known to display normal locomotion (Chiamulera et al., 2001), this effect could be attributed to a reduced exploratory activity. Unlike WT control, Grm5-/- mice explored more actively the novel conspecific during the last $5 \mathrm{~min}$ of the test $[0-5$ vs. $5-10$ min: WT, paired $t$-test: $t(1,15)=0.25$, $p=0.79$; Grm5-/-: $t(1,15)=2,68, p<0.05]$ (Figures 1G,H). Grm5-/- mice also exhibited a longer latency to explore the side chambers of the apparatus at the beginning of the test [ $t$-test: $t(1,30)=4.39, p<0.001$ ] (Figure 1I). These findings suggest that gene-targeted deletion of Grm5 leads to enhanced social interaction, as measured by the higher social preference index, but also to a reduced exploratory activity or enhanced anxiety 

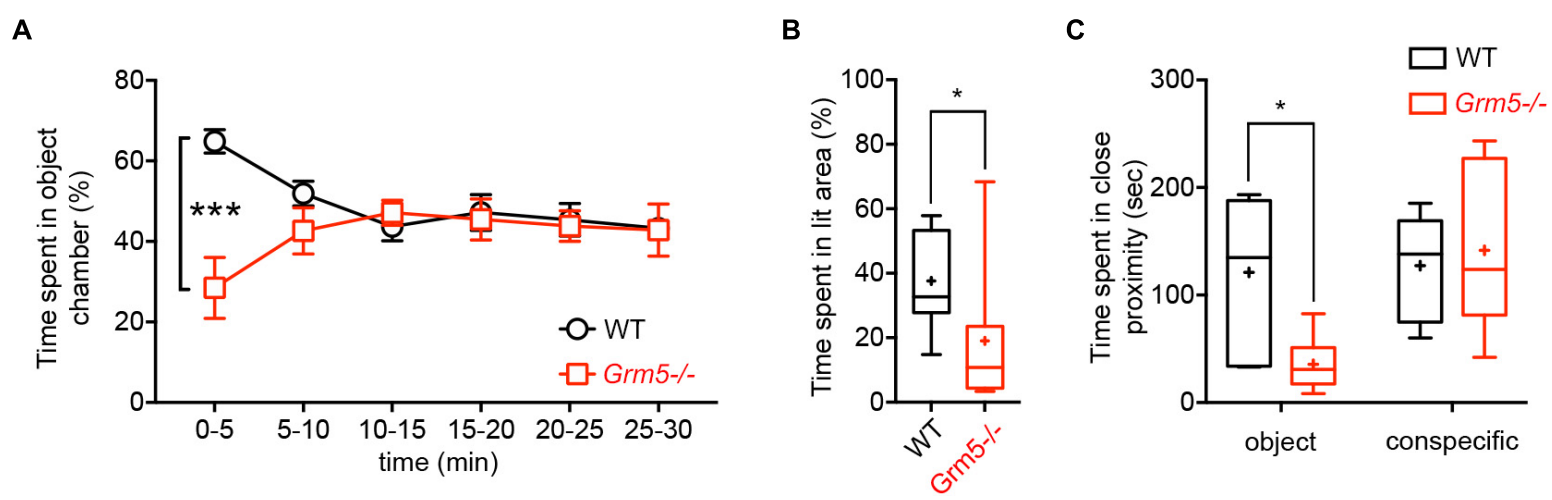

FIGURE 2 | Effects of mGlu5 receptor ablation on non-social exploration and anxiety-like behavior. (A) Time spent in object chamber (\%) by WT and Grm5-/- mice during a 30 min object interaction test in the three-chambered apparatus ( $n=9 /$ genotype). Points represent mean \pm SEM. (B) Time spent in the lit area (\%) by WT and Grm5-/- mice in a Light-dark Test ( $n=12 /$ genotype). (C) Time spent in close proximity to an object (empty mesh cylinder) or to an encaged conspecific during the Forced Social or Object interaction test ( $n=6-7 /$ group). Boxplots represent median, upper and lower quartiles with 10 th and 90 th percentile whiskers. Mean is represented with a cross. ${ }^{*} p<0.05,{ }^{* *} p<0.01,{ }^{* * *} p<0.001$.

as suggested by the reduced distance traveled and high latency to explore the side chambers. To assess whether the lower time spent in the object chamber was due to the putative anxiogenic phenotype or an exploration deficit, we performed an additional experiment in which mice were presented only with the novel object (i.e., the empty enclosure), while the opposite chamber of the apparatus was left empty. In the first $5 \mathrm{~min}$ of a $30 \mathrm{~min}$ session, Grm5-/- indeed explored significantly less the object chamber than WT mice [2-way repeated measures ANOVA: time $F(5,85)=0.36$; genotype $F(1,17)=3.66$; time $\times$ genotype $F(5,85)=7.38 p<0.001 ; 5$ min: WT vs. Grm5-/-: $p<0.001]$ (Figure 2A). Conversely, in the remaining time of the session the two genotypes showed no difference in time spent in the object chamber (Figure 2A), therefore, showing no generalized deficit in exploration. These findings strongly suggest that Grm5-/- mice have an axiogenic phenotype.

Thus, we specifically tested these mice for measures of anxiety-like behavior using the light-dark-box test. Grm5-/mice spent indeed significantly less time in the bright side of the box as compared to WT mice $[t$-test: $t(1,22)=2.42$, $p<0.05$ ] (Figure 2B).

Taken together, these results indicate that Grm5-/- mice show an anxiogenic phenotype although with a paradoxically enhanced sociability as measured with social ratios.

\section{Pharmacological Blockade of mGlu5 Receptors Is Anxiolytic Without Affecting Sociability}

We next assessed the effects of mGlu5 receptor negative allosteric modulation in WT C57BL/6J mice in sociability and anxiety. We assessed the effects of MTEP (10 mg/kg) at two different time points, when MTEP receptor occupancy should be at its peak (5-15 min post-i.p. injection) and when it should have returned at baseline levels ( $1 \mathrm{~h}$ post-i.p. injection) (Anderson et al., 2003). Vehicle- and MTEP-treated animals displayed sociability, spending more time in the conspecific chamber than in the novel object chamber both at $5 \mathrm{~min}$ [2-way ANOVA: chamber $F(2,72)=233, p<0.001$; treatment $F(1,72)=0.002, p>0.05$; and chamber $\times$ treatment $F(2,72)=4.58, p<0.05$; object chamber vs. conspecific chamber: WT $p<0.001$, Grm5-/$p<0.001$ ] and $1 \mathrm{~h}$ post-injection [2-way ANOVA: chamber $F(2,66)=196.8, p<0.001$; treatment $F(1,66)=0.001, p>0.05$; chamber $\times$ treatment $F(2,66)=0.59, p>0.05$ ] (Figures 3A,G). MTEP had no effects on the overall time spent in the novel conspecific or novel object chamber either at $5 \mathrm{~min}$ (WT vs. Grm5-/-: $p>0.05$ ) or at $1 \mathrm{~h}$ post-injection (WT vs. Grm5-/-: $p>0.05$ ) (Figures 3A,G). However, MTEP reduced the amount of time spent interacting closely with the conspecific and object in a non-specific manner at 5 min [2-way ANOVA: treatment $F(1,48)=13.6, p<0.05$; close interaction $F(1,48)=84.9$, $p<0.05$; treatment $\times$ close interaction $F(1,48)=1,93, p=0.17]$ (Figure 3B) and at $1 \mathrm{~h}$ post-injection [2-way ANOVA: treatment $F(1,44)=6.14, p<0.05$; close interaction $F(1,44)=54.8$, $p<0.05$; treatment $\times$ close interaction $F(1,44)=0.53, p=0.47]$ (Figure $3 \mathbf{H})$. Social preference indexes for both conspecific chamber time and time in close interaction with the conspecific did not differ between vehicle and MTEP-treated animals both at $5 \mathrm{~min}$ [chamber: $t$-test: $t(1,24)=0.102, p>0.05$; close interaction: $t$-test: $t(1,24)=0.312, p>0.05]$ (Figures 3C,D) and $1 \mathrm{~h}$ post-injection [chamber: $t$-test: $t(1,22)=0.95, p>0.05$; close interaction: $t$-test: $t(1,22)=0.36, p>0.05$ ] (Figures 3I,J). These findings indicate that the interaction with a conspecific is not altered by acute MTEP treatment. The lowered active exploration of the conspecific in mice treated with MTEP was accompanied by a marked decrease in object exploration and an increase in locomotor activity during the test, as shown by the distance traveled during the test at $5 \mathrm{~min}$ post-injection [ $t$ test: $t(1,24)=4.56, p<0.001$ ] (Figure 3E) as well as by a statistical trend toward significance at $1 \mathrm{~h}$ post injection [t-test: $t(1,22)=1.77, p=0.09$ ] (Figure 3K).

We then tested whether the reduced active exploration of the object and conspecific during the test was due to the proposed anxiolytic activity of MTEP (Klodzinska et al., 2004; 

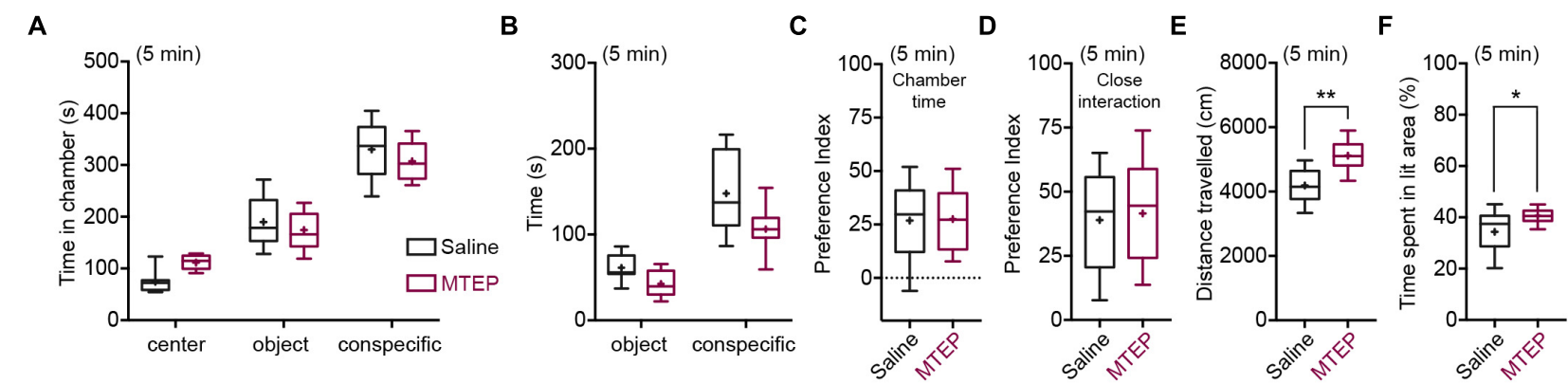

G

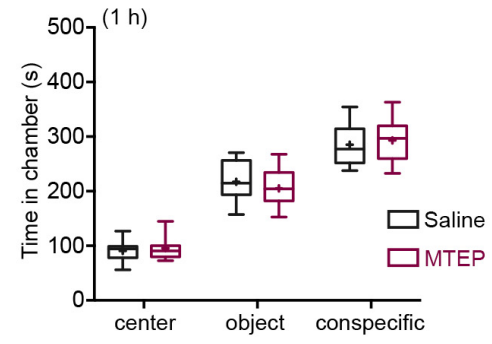

H

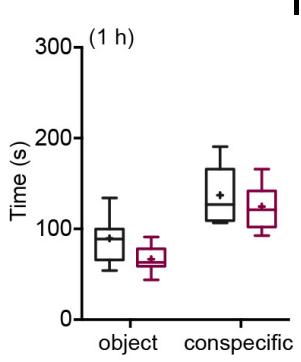

I

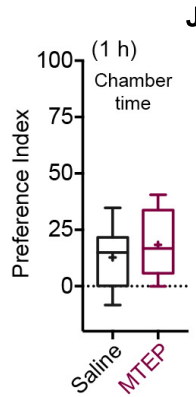

J

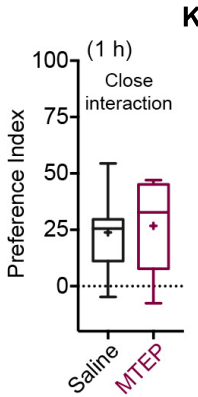

K

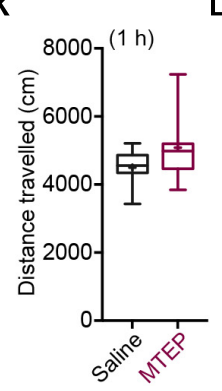

$\mathbf{L}$

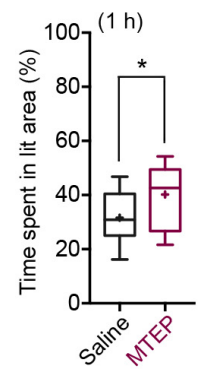

FIGURE 3 | Pharmacological blockade of mGlu5 receptors is anxiolytic without affecting sociability. Time spent (s) in the different compartments of the three-chambered apparatus by MTEP- and vehicle-treated (4\% DMSO in saline) mice during the test, at 5 min (MTEP $n=13$; vehicle $n=13$ ) (A) or $1 \mathrm{~h}$ post-injection (MTEP $n=12$; vehicle $n=12)(\mathbf{G})$. Time spent (s) in close proximity with the conspecific and object at 5 min $\mathbf{( B )}$ or $1 \mathrm{~h}$ post-injection $\mathbf{( H )}$. Preference index derived from the numerical difference between time spent in conspecific and object chamber divided by total time spent $\times 100$ at 5 min (C) or $1 \mathrm{~h}$ post-injection (I). Index derived from the numerical difference between time spent in close proximity to conspecific and object divided by total time spent in close proximity $\times 100$ at 5 min (D) or $1 \mathrm{~h}$ post-injection (J). Both social preference indexes did not differ between MTEP and vehicle-treated mice at any post-injection time. At 5 min (E) post-injection, but not at $1 \mathrm{~h}(\mathbf{K})$, MTEP-treated mice traveled a longer distance than vehicle-treated mice. In the light-dark test, both at 5 min (MTEP $n=13$; vehicle $n=13)(\mathbf{F})$ and $1 \mathrm{~h}($ MTEP $n=14$; vehicle $n=14)(\mathbf{L})$ post-injection, mice treated with MTEP displayed anxiolytic-like activity, spending more time (\%) in the lit area of the box than vehicle-treated mice. Boxplots represent median, upper and lower quartiles with 10th and 90th percentile whiskers. Mean is represented with a cross. $* p<0.05, * * p<0.01$

Stachowicz et al., 2007; Lee et al., 2017, 2018; but see Ade et al., 2016). Similar to sociability, we tested the effects of MTEP at both 5 and $1 \mathrm{~h}$ post-injection in the light-dark test. MTEP-treated animals spent significantly more time in the bright side of the box, both at $5 \min [t(1,24)=2.55, p<0.05]$ (Figure 3F) and at $1 \mathrm{~h}$ post-injection $[t(1,26)=2.08, p<0.05]$ (Figure $3 \mathrm{~L}$ ). Taken together, these results confirm the anxiolytic action of MTEP and suggest that mGlu5 receptor NAM does not influence sociability in WT mice when administered acutely.

\section{Aberrant Brain Region-Specific Activation After Social Exposure in Grm5-/- Mice}

In order to investigate whether the increased sociability observed in Grm5-/- mice could result from different patterns of brain activation upon exposure to social and non-social cues, we exposed WT and Grm5-/- mice in their home cage to either a novel conspecific or novel object and quantified the expression of the immediate early gene c-Fos. We used three independent experimental groups for each genotype: a group exposed to an age-matched conspecific enclosed into a wire mesh cage (conspecific group), a group exposed only to the wire mesh cage (object group), and a third control group left undisturbed in the home cage $(\mathrm{HC})$. Similar to the threechambered social task, Grm5-/- mice displayed a reduced exploration of the novel object in comparison to WT [2way ANOVA: cue $F(1,22)=6.05$; genotype $F(1,22)=2.45$; cue $\times$ genotype $F(1,22)=4.80, p<0.05$; WT vs. Grm5-/object: $p<0.05$ ] and a similar exploration of the novel conspecific (Figure 2C). Two hours after the exposure, mice were perfused and processed for immunocytochemistry. Twenty-one brain regions, previously reported to be activated after social interaction (Kim et al., 2015), were preselected for c-Fos analysis. In mice kept undisturbed in their home cage, no significant differences between WT and Grm5-/- mice were detected in the number of c-Fos+ neurons in any of the areas analyzed (see Table 1 for statistical significance, Figure 4 and Supplementary Figure S1). This suggests that under resting conditions basal activity in the set of brain areas that we have analyzed is not altered by the lack of mGlu5 receptors. Conversely, compared to the HC group a significant increase in the number of c-Fos+ cells was observed in most of the areas analyzed with the exception of AcbC, LSI, CA1, CA2, and PMCo in WT mice after exposure to a novel object; AcbC, LSI, CA2, and PMCo in WT mice after exposure to a conspecific and AcbC, LSI, CA1, and PMCo in Grm5-/-mice after exposure to a novel object (see Table 1). 


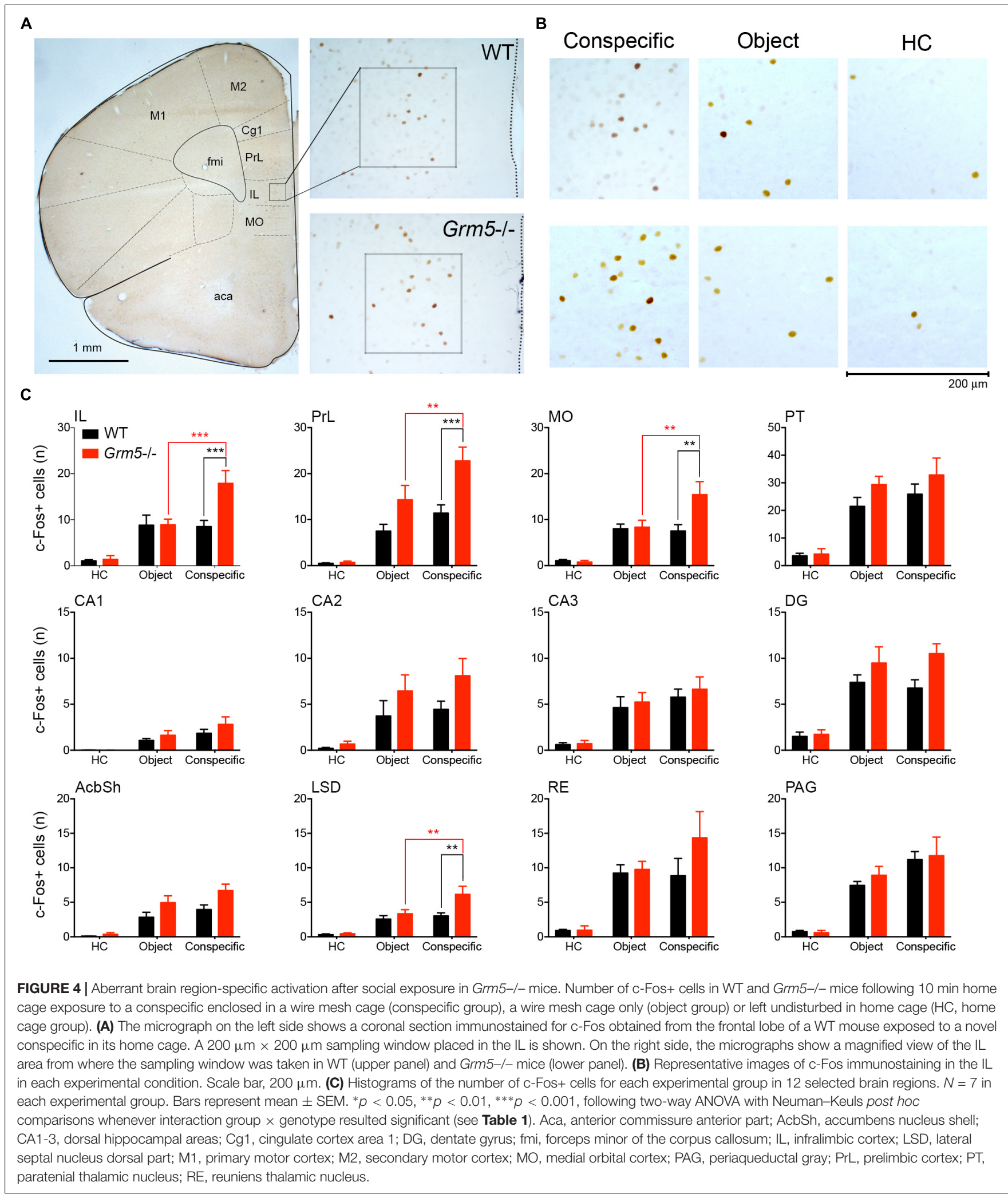

A significant group $\times$ genotype interaction in the 2-way ANOVAs was observed only in 4 brain areas, namely IL, MO, PrL, and LSD (see Table 1 and Figure 4). The interaction with a conspecific triggered higher activation as compared with the object in Grm5-/- mice, whereas no statistical significant differences were observed between object and conspecific 


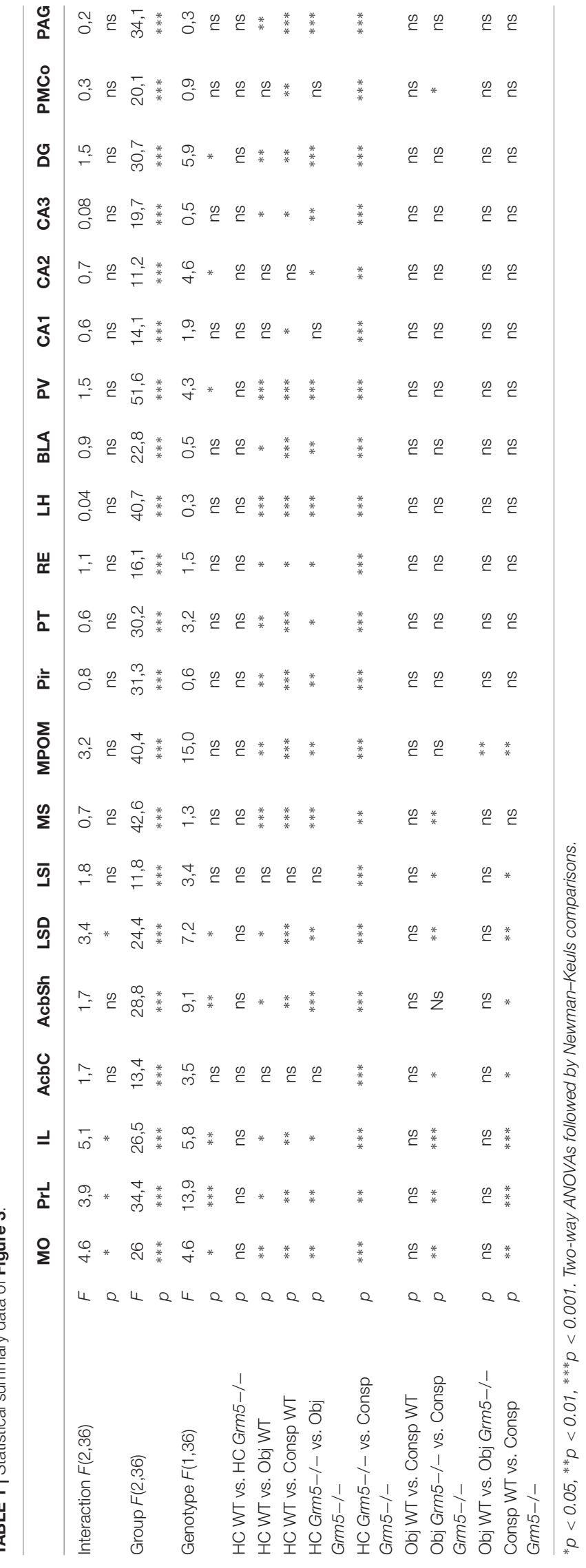

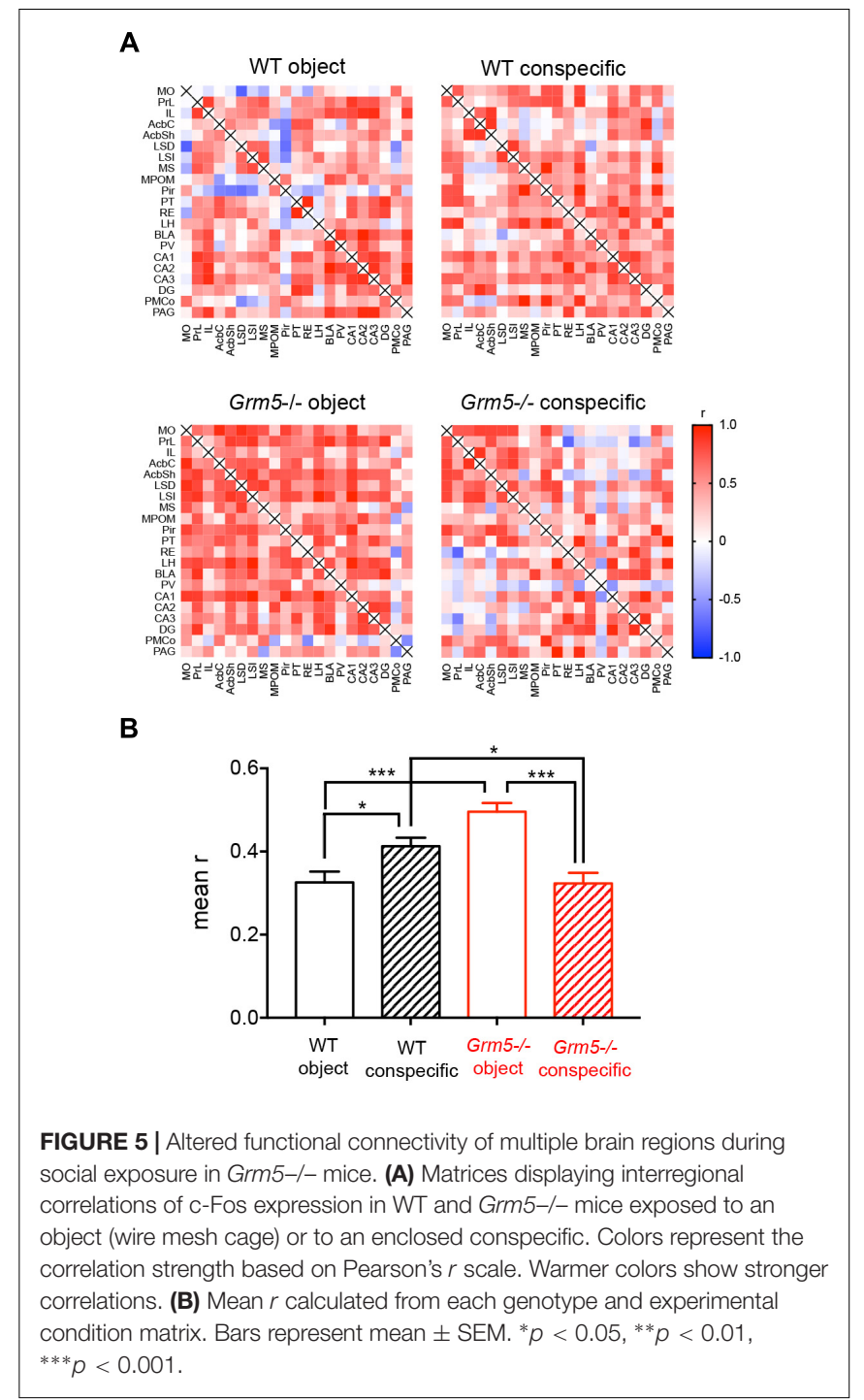

exposed-groups in WT mice. These four brain areas had also a higher number of c-Fos+ cells in Grm5-/- mice as compared to WT mice when exposed to the novel conspecific, but not to the novel object (see Table 1 and Figure 4). The arousal produced by the exposure to the novel object or conspecific in our paradigm may have masked in WT mice distinct pattern of c-Fos activation. On the other hand, the lack of mGlu5 receptors was able to induce region-specific changes in the number of c-Fos+ cells specifically related to social interaction. We, thus, reasoned that social interaction, contrary to nonsocial, rather than producing a higher degree of activation, namely number of c-Fos + cells per area, it enhances functional connectivity among a set of brain regions. In addition, the high activation of prefrontal areas and LSD observed in Grm5-/mice, could underlie a disrupted activity coordination. To explore this possibility, we analyzed the functional connectivity generated during social or object investigation in WT and Grm5-/- mice. 


\section{Altered Functional Connectivity of Multiple Brain Regions During Social Exposure in Grm5-/- Mice}

In order to infer interactions between neural elements, we computed correlation coefficients across subjects using our c-Fos expression data set (Horwitz et al., 1995; Tanimizu et al., 2017a,b; Rogers-Carter et al., 2018). This allowed us to obtain an approximation of the strength of the coordinated activity changes among brain regions following social and non-social interactions in Grm5-/- and WT mice. We first computed interregional correlations for each experimental group (Figure 5A). As shown in Figure 5B, changes in network density upon social investigation were observed both in WT and Grm5-/- mice [2-way ANOVA: genotype $F(1,836)=6.50, p<0.05$; group $F(1,836)=6.66, p<0.01$; group $\times$ genotype $F(1,836)=47.51$, $p<0.001]$. A higher functional connectivity (mean $r$ ) was observed upon conspecific as compared to object interaction in WT mice $(p<0.05)$. Conversely, in Grm5-/- mice functional connectivity was higher upon non-social interaction $(p<0.001)$. Interaction with a conspecific led to higher functional connectivity in WT mice as compared to Grm5-/mice $(p<0.05)$.

These results suggest that in WT mice social investigation leads to a higher functional connectivity than upon interaction with an object. On the contrary, the lack of mGlu5 receptors inverts the strength of the functional connectivity toward nonsocial interaction.

\section{Social Interaction Network Hubs Are Rearranged in Grm5-/- Mice}

Based on interregional matrices (Figure 5A), we generated network graphs (Figure 6A), where nodes represent brain regions and edges represent above-threshold (Pearson's $r>0.6$ ) significant $(p<0.05)$ correlations (Figure 6A). We further applied graph theoretical analysis to the network graphs to explore if social investigation induces changes to the relative weight of any of the identified nodes and whether they are affected by the lack of mGlu5 receptors (Figure 6B). We then computed the within-community $z$ score and participation coefficient for each node (Figure 6C). In correlation-based networks, the within-community $z$ score measures how well connected a region is to its own community and the participation coefficient of a node constitutes a measure of the degree to which a node is linked with nodes in other communities (Guimerà and Amaral, 2005). The participation coefficient, therefore, denotes "hubness" (Power et al., 2013; Rogers-Carter et al., 2018). Nodes displaying a high value of within-community $z$ score and participation coefficient are thought to be key hubs, crucial for coordinating other nodes and, thus, the overall activity of the network (Tanimizu et al., 2017a). Our analysis identified the RE, AcbSh, PAG, and CA3 as key hubs in the social interaction functional network and the PT in the object functional network in WT mice. In addition, the hippocampal regions CA1, CA2, and DG as well as the IL appear as key regions in coordinating the activity between communities in the social interaction network given their high participation coefficient.
Of note is the differential participation of the PrL and IL in the object and conspecific functional networks, respectively. In Grm5-/- mice, we observed a complete derangement in the role of nodes in both the non-social and social functional networks. In these animals, while the hippocampus and mPFC lost their coordinating role in conspecific network activity, the lateral septum increased it. Moreover, the PT transferred its role as a key hub from the non-social to the social functional network.

\section{DISCUSSION}

Here, we reassessed how genetic ablation and pharmacological blockade of mGlu5 receptors affect sociability and anxiety-like behavior in mice. We show that germ line deletion of mGlu5 receptors leads to an anxiogenic phenotype accompanied by a paradoxical enhancement of sociability. Conversely, negative allosteric modulation of this receptor reduced anxiety-like behavior in the light-dark test, consistent with previous studies (Klodzinska et al., 2004; Stachowicz et al., 2007; Lee et al., 2017, 2018), without influencing sociability in WT mice. We further determined how the lack of mGlu5 receptors affects the pattern of brain activation following social and non-social interaction by quantifying the number of neurons expressing the IEG c-Fos. A computational approach was then used to model the potential impact of mGlu5 receptor ablation on functional connectivity of brain areas relevant for social interaction. Our c-Fos quantification revealed a restricted activation, limited to the MO, PrL, IL, and LSD, in Grm5-/- mice following interaction with a conspecific as compared to a novel object, although the anxiogenic phenotype of these animals could have in part influenced our analysis. On the other hand, the lack of wideranging changes in cFos expression between social and non-social interactions in control mice might have been confounded by the high arousal state induced by the alteration of the home cage environment. Alternatively, our computational analysis suggests that social interaction, rather than inducing broad changes in c-Fos expression, leads to an increased functional connectivity of specific brain regions important for social behavior.

As a first step toward understanding the role of mGlu5 receptors in social preference, we examined the behavior of Grm5-/- mice in the three-chambered social task. We observed that ablation of the mGlu5 receptor enhances sociability, as indicated by the social preference index. This was accompanied by an increase in anxiety-like behavior, that was initially observed as a delayed exploration of the side chambers and a reduced distance traveled in the three-chambered social task. This was then confirmed by the light-dark test in which Grm5-/- mice spent less time in the lit compartment than WT mice. The anxiogenic phenotype observed in Grm5-/- mice appears at odds with the widely accepted anxiolytic action of mGlu5 receptor antagonists (see for review: Ferraguti, 2018) and the reduced stress-induced hyperthermia previously reported for these mice (Brodkin et al., 2002). Grm5-/- mice were previously shown to explore more the center of a novel arena in the open field test, but were found to behave similarly to control animals in the light-dark and elevated plus-maze tests (Olsen et al., 2010). 
A

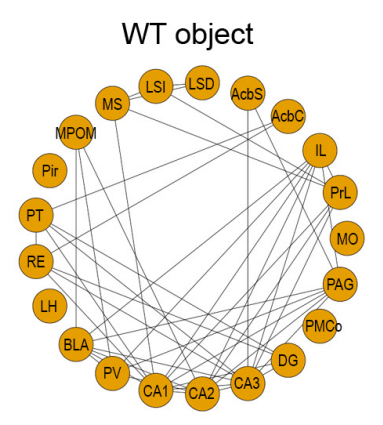

B
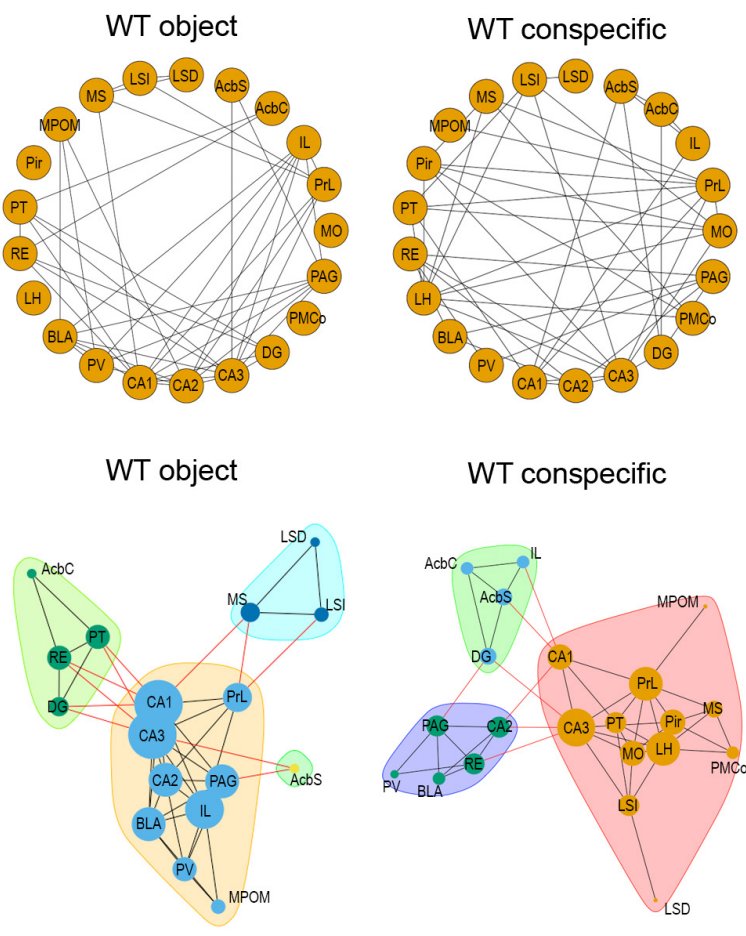

WT conspecific
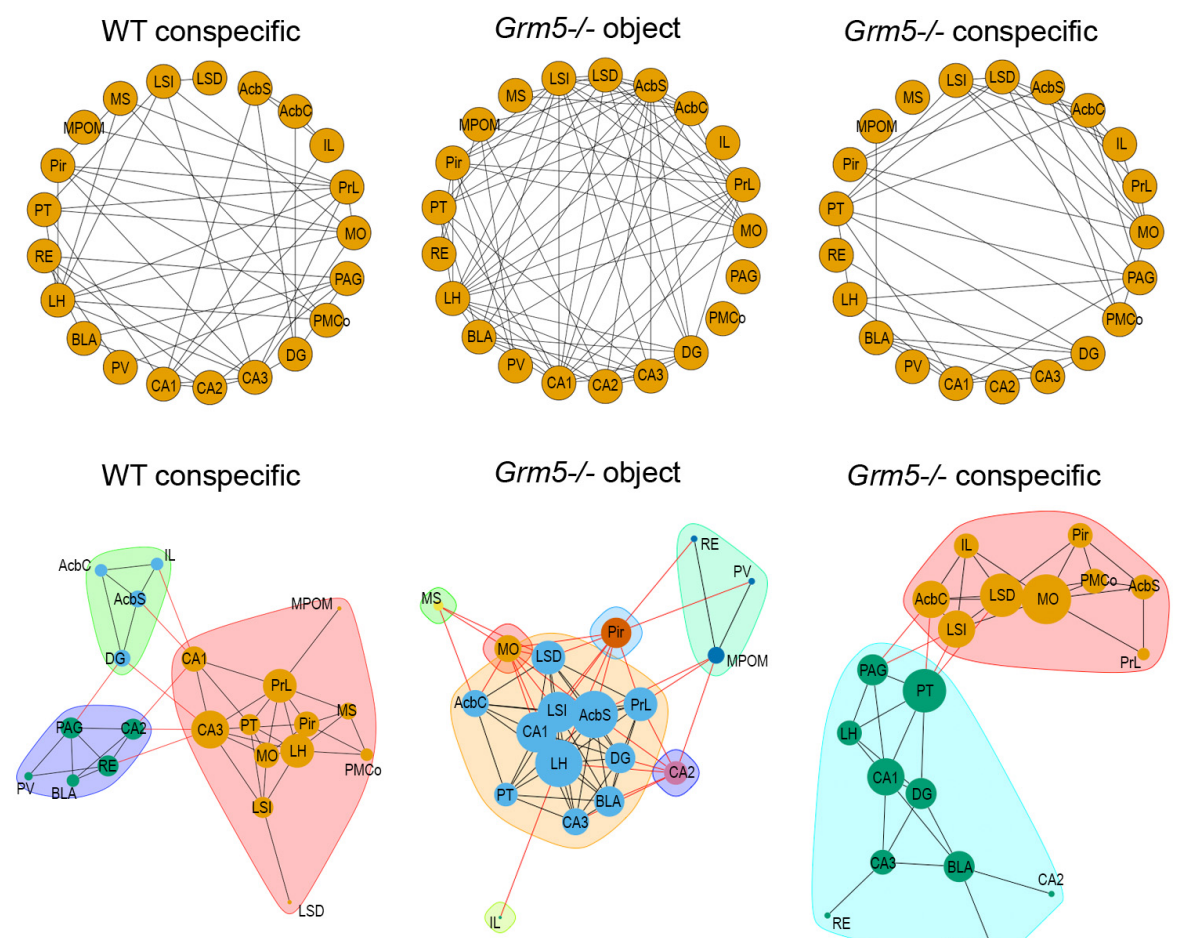

Grm5-/- object

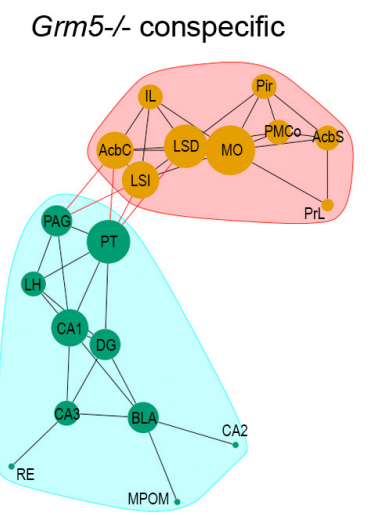

C

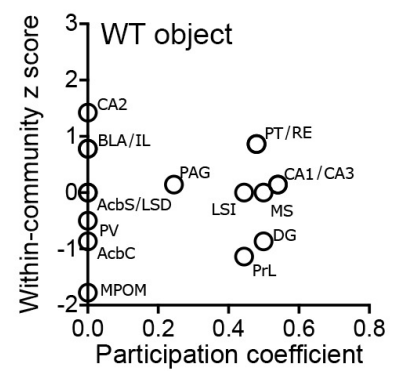

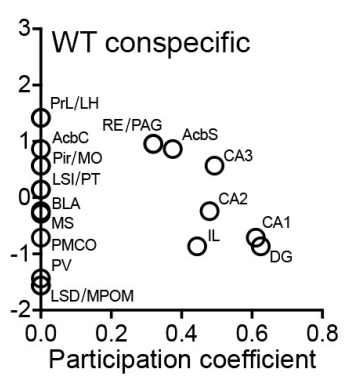

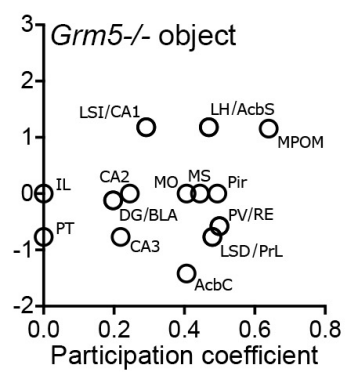

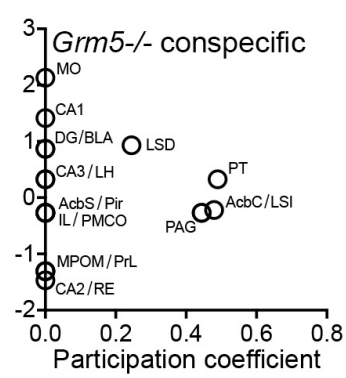

FIGURE 6 | Social interaction network hubs are rearranged in Grm5-/- mice. (A) Network graphs showing above-threshold ( $r>0.6)$ significant positive correlations $(p<0.05)$ between the different brain regions in each experimental condition and genotype. (B) Networks identified for each experimental condition and genotype. The width of the edges is proportional to the correlation strength of above-threshold significant correlations and the node size is proportional to the degree (number of edges associated with a given node) of the node in a given network. Colors in the network represent the different communities identified via modularity optimization. (C) Within-community z scores and participation coefficients of each brain region in the different networks generated following interaction with an object or conspecific in WT and Grm5-/- mice.

However, Inta et al. (2013) reported an age-dependent anxiogenic phenotype in Grm5-/ - mice using the light-dark test, consistent with our findings. These controversies could be explained by differences in the anxiety tests used, e.g., conflict-based vs. physiological measurement, or by procedural variations. Since Grm5-/- mice have a normal locomotor activity (Chiamulera et al., 2001), we can exclude that the reduced time spent in the lit compartment of the light-dark test could have resulted from a motor impairment.

Inhibition of mGlu5 receptor activity through systemic administration of antagonists was found to rescue the impaired social behavior typical of the BTBR inbred mouse strain and of different mouse models of ASD (Silverman et al., 2012;
Gantois et al., 2013; Chung et al., 2015). On the other hand, inconsistent effects of mGlu5 antagonists were reported in WT rodents, e.g., MTEP induced social isolation in rats (Koros et al., 2007), whereas MPEP increased sniffing and extended time spent interacting in Balb/c mice, but reduced sniffing in Swiss Webster mice (Burket et al., 2011).

In our study, the three-chambered social task revealed increased social preference in Grm5-/- mice, based on the preference index, despite the total time spent in the social chamber was similar to that of WT animals. However, given the increased anxiety-like behavior showed by Grm5-/- mice, the sociability expressed by these animals in the three-chambered social task might have been underestimated. Selective ablation of 
the mGlu5 receptor in parvalbumin-positive neurons resulted in higher duration of social interaction bouts (Barnes et al., 2015), whereas their ablation in cortical glutamatergic principal cells did not produce any detectable effect (Jew et al., 2013). The increased sociability in germline Grm5-/ - mice may, therefore, primarily result from a role of mGlu5 receptors at inhibitory circuits. Further studies should explore the pathways and neurons at which mGlu5 receptors regulate sociability.

Our pharmacological study shows that negative allosteric modulation of mGlu5 receptors with MTEP had no effect on sociability despite it reduced active exploration of both the conspecific and object. These non-specific effects of MTEP on social and non-social interactions could explain the reported social isolation in rats after MTEP treatment (Koros et al., 2007). The complex effects on measures of sociability observed in mice (Burket et al., 2011), on the other hand, may depend on the known off-target effects of MPEP (Lea and Faden, 2006). The anxiolytic action elicited by MTEP in WT mice in our study, although modest, confirms that the absence of an effect on sociability does not result from a lack of activity of the drug.

Both mGlu5 receptor NAMs MPEP and MTEP have been described as potent anxiolytics in different rodent models (see for review: Ferraguti, 2018). However, their anxiolytic properties on WT mice appear to greatly depend on strain, dose and delay between administration and testing. MTEP was found to be anxiolytic at $3 \mathrm{mg} / \mathrm{kg}$ and anxiogenic at $30 \mathrm{mg} / \mathrm{kg}$ in the lightdark test in C57BL/6j (Lee et al., 2018). Whether this differential effect on anxiety is due to an inverted U-shaped dose-response activity or to potential unspecific effects of the highest dose of MTEP remains to be explored.

From these findings, it could be concluded that deviations in any direction of mGlu5 receptor function may lead to impairments in both social and anxiety-like behaviors.

To understand at the anatomical level where mGlu5 receptors regulate brain activity during social exploration, we have analyzed the expression of c-Fos in a selected set of brain areas previously reported to be activated upon social interaction (Kim et al., 2015). Our study shows that in Grm5-/- mice, MO, PrL, IL, and LSD were selectively activated upon interaction with a conspecific, suggesting that mGlu5 receptors dampen neuronal activity in these brain regions during social behavior, possibly by activating interneurons and facilitating feedforward inhibition (Pollard et al., 2014). Future studies will have to unveil the expression and role of mGlu5 receptors in the different neuronal types in these brain areas and their specific contribution to social behavior.

Our functional connectivity analysis of 21 brain regions relevant for social behavior suggested a disruption of the network density in Grm5-/- during exploration of both social and non-social stimuli. Moreover, the networks generated during social and non-social interactions, as well as the role of individual brain regions in coordinating network activity, such as hippocampal and prefrontal areas, dramatically changed in Grm5-/- mice. In line with previous findings investigating prolonged social interactions (Tanimizu et al., 2017a), we observed high participation coefficients of the hippocampus and $\mathrm{mPFC}$ in WT mice exposed to a conspecific. It should be taken into account, however, that c-Fos correlation-based networks suffer from several limitations. Inclusion of different brain regions into the network can lead to rearrangements of the communities, measures of centrality and roles of individual nodes. Thus, with current computational models it is hard to compare the role of single nodes between differently generated networks or even between similar experiments that include different brain regions into the network. Nonetheless, c-Fos based functional connectivity and network analysis can serve as a promising tool for hypothesis generation (Vetere et al., 2017; Rogers-Carter et al., 2018), although promising key hubs will have to be validated experimentally, e.g., using chemo- or opto-genetic approaches.

The hippocampus and mPFC, together with the amygdala, appear as key regions underlying sociability circuits (Felix-Ortiz and Tye, 2014; Felix-Ortiz et al., 2016). The CA2 region, in particular, has been recently proposed as a critical hub for socio-cognitive memory processing (Hitti and Siegelbaum, 2014), independent of other hippocampus-dependent behaviors such as spatial memory. In our study, the CA2 region was shown to display largely different roles in the functional networks generated following a 10 min interaction with a conspecific or an object, sufficient time to allow for social and non-social memory formation (Tanimizu et al., 2017a,b). In the network generated upon interaction with a conspecific in Grm5-/- mice, the CA2 region was the most isolated node in the network. Therefore, it would be of interest to determine whether manipulation of mGlu5 receptors activity in this region affects social recognition memory as predicted by our functional network analysis.

To our knowledge, only one study has attempted to address the contribution of mGlu5 receptors in a specific brain region to sociability, so far. In line with our findings that the lack of mGlu5 receptors increases activity in the LSD upon social interaction, Mesic et al. (2015) suggested that selective removal of mGlu5 receptors in this brain area impaired expression of sociability but not social novelty.

In conclusion, our work shows that while the mGlu5 receptor NAM MTEP is anxiolytic upon systemic acute administration, the lack of these receptors, as in germ line Grm5-/- mice, results in an anxiogenic phenotype. Similarly, while sociability is not affected by pharmacological blockade of mGlu 5 receptors, their lack leads to an apparent increased sociability. Whether this is the result of complex and perhaps opposite effects at different brain regions or developmental adaptations remains to be established. Indeed, a further note of caution concerns data obtained with germ line knockouts as adaptive changes may influence behavior differently from manipulations carried out in adult animals. Further studies in which mGlu5 receptor activity is abolished or modulated in a time-controlled and region- and/or cell-type specific manner are, therefore, warranted. Our c-Fos expression and network analyses offers candidate areas for such specific targeting.

Taken together our findings support the relevance of mGlu5 receptors in modulating anxiety-like behavior and sociability. The paradoxical increased social preference within an overall anxiogenic phenotype, as in the germ line Grm5-/- mice, shows remarkable analogies with Williams syndrome (MeyerLindenberg et al., 2006; Barak and Feng, 2016). Future studies 
should address whether mGlu5 function is altered in this rare neurodevelopmental disorder extending the implication of these receptors beyond $\mathrm{ASD}$.

\section{AUTHOR CONTRIBUTIONS}

AR-P and FF conceived and designed the project. AR-P, JK, MZ, EP, and GS were involved with experimental and analytical aspects of the manuscript. AR-P performed data analyses, functional connectivity, and graph theoretical analysis. AR-P and FF wrote the manuscript. All contributing authors commented on the manuscript.

\section{FUNDING}

We acknowledge financial support from the Austrian Science Fund (Fonds zur Förderung der Wissenschaftlichen Forschung) grant W12060-B10 to FF.

\section{REFERENCES}

Ade, K. K., Wan, Y., Hamann, H. C., O’Hare, J. K., Guo, W., Quian, A., et al. (2016). Increased metabotropic glutamate receptor 5 signaling underlies obsessivecompulsive disorder-like behavioral and striatal circuit abnormalities in mice. Biol. Psychiatry 80, 522-533. doi: 10.1016/j.biopsych.2016.04.023

Allsop, S. A., Vander Weele, C. M., Wichmann, R., and Tye, K. M. (2014). Optogenetic insights on the relationship between anxiety-related behaviours and social deficits. Front. Behav. Neurosci. 8:241. doi: 10.3389/fnbeh.2014.00241

Anderson, J. J., Bradbury, M. J., Giracello, D. R., Chapman, D. F., Holtz, G., Roppe, J., et al. (2003). In vivo receptor occupancy of mGlu5 receptor antagonists using the novel radioligand $[3 \mathrm{H}] 3$ methoxy-5-(pyridin-2-ylethynyl)pyridine). Eur. J. Pharmacol. 473, 35-40. doi: 10.1016/S0014-2999(03)01935-6

Barak, B., and Feng, G. (2016). Neurobiology of social behavior abnormalities in autism and Williams syndrome. Nat. Neurosci. 19, 647-655. doi: 10.1038/nn. 4276

Barnes, S. A., Pinto-Duarte, A., Kappe, A., Zembrzycki, A., Metzler, A., Mukamel, E. A., et al. (2015). Disruption of mGluR5 in parvalbumin-positive interneurons induces core features of neurodevelopmental disorders. Mol. Psychiatry 20, 1161-1172. doi: 10.1038/mp.2015.113

Berry-Kravis, E., Des Portes, V., Hagerman, R., Jacquemont, S., Charles, P., Visootsak, J., et al. (2016). Mavoglurant in fragile X syndrome: results of two randomized, double-blind, placebo-controlled trials. Sci. Transl. Med. 8:321ra5. doi: 10.1126/scitranslmed.aab4109

Bhakar, A. L., Dölen, G., and Bear, M. F. (2012). The pathophysiology of fragile $\mathrm{X}$ (and what it teaches us about synapses). Annu. Rev. Neurosci. 35, 417-443. doi: 10.1146/annurev-neuro-060909-153138

Brodkin, J., Bradbury, M., Busse, C., Warren, N., Bristow, L. J., and Varney, M. A. (2002). Reduced stress-induced hyperthermia in mGluR5 knockout mice. Eur. J. Neurosci. 16, 2241-2244. doi: 10.1046/j.1460-9568.2002.02294.x

Brody, S. A., Conquet, F., and Geyer, M. A. (2004). Effect of antipsychotic treatment on the prepulse inhibition deficit of mGluR5 knockout mice. Psychopharmacology 172, 187-195. doi: 10.1007/s00213-003-1635-3

Brody, S. A., and Geyer, M. A. (2004). Interactions of the mGluR5 gene with breeding and maternal factors on startle and prepulse inhibition in mice. Neurotox. Res. 6, 79-90. doi: 10.1007/BF03033300

Burket, J. A., Herndon, A. L., Winebarger, E. E., Jacome, L. F., and Deutsch, S. I. (2011). Complex effects of mGluR5 antagonism on sociability and stereotypic behaviors in mice: possible implications for the pharmacotherapy of autism spectrum disorders. Brain Res. Bull. 86, 152-158. doi: 10.1016/j.brainresbull. 2011.08.001

\section{ACKNOWLEDGMENTS}

We are grateful to Sabine Schönherr for excellent technical assistance. We also thank Enrica Paradiso for reading and commenting a previous version of the manuscript.

\section{SUPPLEMENTARY MATERIAL}

The Supplementary Material for this article can be found online at: https://www.frontiersin.org/articles/10.3389/fnmol. 2019.00030/full\#supplementary-material

FIGURE S1 | Histograms of the number of c-Fos+ cells for each experimental group in the other 9 selected brain regions. $N=7$ in each experimental group. Bars represent mean \pm SEM. ${ }^{*} p<0.05,{ }^{* *} p<0.01,{ }^{* * *} p<0.001$, following two-way ANOVA with Neuman-Keuls post hoc comparisons whenever interaction group $\times$ genotype resulted significant (see Table 1). AcbC, accumbens nucleus core; BLA, basolateral amygdala; LH, lateral hypothalamic area; LSI, lateral septal nucleus intermedial part; MPOM, medial preoptic nucleus medial part; MS, medial septal nucleus; Pir, piriform cortex; PMCo, posteromedial cortical amygdaloid area; PV, paraventricular thalamic nucleus.

Busse, C. S., Brodkin, J., Tattersall, D., Andersen, J. J., Warren, N., Tehrani, L., et al. (2004). The behavioral profile of the potent and selective mGlu5 receptor antagonist 3-[(2-methyl-1,3-thiazol-4-yl)ethynyl] pyridine (MTEP) in rodent models of anxiety. Neuropsychopharmacology 29, 1971-1979. doi: 10.1038/sj. npp. 1300540

Chiamulera, C., Epping-Jordan, M. P., Zocchi, A., Marcon, C., Cottiny, C., Tacconi, S., et al. (2001). Reinforcing and locomotor stimulant effects of cocaine are absent in mGluR5 null mutant mice. Nat. Neurosci. 4, 873-874. doi: 10.1038/ nn0901-873

Chung, W., Choi, S. Y., Lee, E., Park, H., Kang, J., Park, H., et al. (2015). Social deficits in IRSp53 mutant mice improved by NMDAR and mGluR5 suppression. Nat. Neurosci. 18, 435-443. doi: 10.1038/nn.3927

Csardi, G., and Nepusz, T. (2006). The igraph software package for complex network research. InterJ. Complex Syst. 1695, 1-9.

D’Antoni, S., Spatuzza, M., Bonaccorso, C. M., Musumeci, S. A., Ciranna, L., Nicoletti, F., et al. (2014). Dysregulation of group-I metabotropic glutamate (mGlu) receptor mediated signalling in disorders associated with Intellectual Disability and Autism. Neurosci. Biobehav. Rev. 46, 228-241. doi: 10.1016/j. neubiorev.2014.02.003

Dölen, G., Osterweil, E., Rao, B. S., Smith, G. B., Auerbach, B. D., Chattarji, S., et al. (2007). Correction of fragile X syndrome in mice. Neuron 56, 955-962. doi: 10.1016/j.neuron.2007.12.001

Felix-Ortiz, A. C., Burgos-Robles, A., Bhagat, N. D., Leppla, C. A., and Tye, K. M. (2016). Bidirectional modulation of anxiety-related and social behaviours by amygdala projections to the medial prefrontal cortex. Neuroscience 321, 197-209. doi: 10.1016/j.neuroscience.2015.07.041

Felix-Ortiz, A. C., and Tye, K. M. (2014). Amygdala inputs to the ventral hippocampus bidirectionally modulate social behaviour. J. Neurosci. 34, 586595. doi: 10.1523/JNEUROSCI.4257-13.2014

Ferraguti, F. (2018). Metabotropic glutamate receptors as targets for novel anxiolytics. Curr. Opin. Pharmacol. 38, 37-42. doi: 10.1016/j.coph.2018.02.004

Ferraguti, F., and Shigemoto, R. (2006). Metabotropic glutamate receptors. Cell Tissue Res. 326, 483-504. doi: 10.1007/s00441-006-0266-5

Franklin, K. B. J., and Paxinos, G. (2007). The Mouse Brain in Stereotaxic Coordinates. San Diego, CA: Academic Press.

Gantois, I., Pop, A. S., de Esch, C. E. F., Buijsen, R. A. M., Pooters, T., GomezMancilla, B., et al. (2013). Chronic administration of AFQ056/Mavoglurant restores social behaviour in Fmr1 knockout mice. Behav. Brain Res. 239, 72-79. doi: 10.1016/j.bbr.2012.10.059

Guimerà, R., and Amaral, L. A. (2005). Cartography of complex networks: modules and universal roles. J. Stat. Mech. 2005:P02001-1-P02001-13. doi: 10.1088/ 1742-5468/2005/02/P02001 
Hermans, E., and Challiss, R. A. (2001). Structural, signalling and regulatory properties of the group I metabotropic glutamate receptors: prototypic family C G-protein-coupled receptors. Biochem. J. 359(Pt 3), 465-484. doi: 10.1042/ bj3590465

Hitti, F. L., and Siegelbaum, S. A. (2014). The hippocampal CA2 region is essential for social memory. Nature 508, 88-92. doi: 10.1038/nature13028

Homayoun, H., and Moghaddam, B. (2010). Group 5 metabotropic glutamate receptors: role in modulating cortical activity and relevance to cognition. Eur. J. Pharmacol. 639, 33-39. doi: 10.1016/j.ejphar.2009.12.042

Horwitz, B., McIntosh, A. R., Haxby, J. V., and Grady, C. L. (1995). Network analysis of brain cognitive function using metabolic and blood flow data. Behav. Brain Res. 66, 187-193. doi: 10.1016/0166-4328(94)00139-7

Inta, D., Vogt, M. A., Luoni, A., Filipović, D., Lima-Ojeda, J. M., Pfeiffer, N., et al. (2013). Significant increase in anxiety during aging in mGlu5 receptor knockout mice. Behav. Brain Res. 241, 27-31. doi: 10.1016/j.bbr.2012.11.042

Jew, C. P., Wu, C. S., Sun, H., Zhu, J., Huang, J. Y., Yu, D., et al. (2013). mGluR5 ablation in cortical glutamatergic neurons increases novelty-induced locomotion. PLoS One 8:e70415. doi: 10.1371/journal.pone.0070415

Kim, Y., Venkataraju, K. U., Pradhan, K., Mende, C., Taranda, J., Turaga, S. C., et al. (2015). Mapping social behaviour-induced brain activation at cellular resolution in the mouse. Cell Rep. 10, 292-305. doi: 10.1016/j.celrep.2014.12.014

Kinney, G. G., Burno, M., Campbell, U. C., Hernandez, L. M., Rodriguez, D., Bristow, L. J., et al. (2003). Metabotropic glutamate subtype 5 receptors modulate locomotor activity and sensorimotor gating in rodents. J. Pharmacol. Exp. Ther. 306, 116-123. doi: 10.1124/jpet.103.048702

Klodzinska, A., Tatarczyńska, E., Chojnacka-Wójcik, E., Nowak, G., Cosford, N. D., and Pilc, A. (2004). Anxiolytic-like effects of MTEP, a potent and selective mGlu5 receptor agonist does not involve GABAA signaling. Neuropharmacology 47, 342-350. doi: 10.1016/j.neuropharm.2004.04.013

Koros, E., Rosenbrock, H., Birk, G., Weiss, C., and Sams-Dodd, F. (2007). The selective mGlu5 receptor antagonist MTEP, similar to NMDA receptor antagonists, induces social isolation in rats. Neuropsychopharmacology 32, 562576. doi: 10.1038/sj.npp.1301133

Lea, P. M. IV, and Faden, A. I. (2006). Metabotropic glutamate receptor subtype 5 antagonists MPEP and MTEP. CNS Drug Rev. 12, 149-166. doi: 10.1111/j.15273458.2006.00149.x

Lee, K. M., Coelho, M. A., Class, M. A., and Szumlinski, K. K. (2018). mGlu5dependent modulation of anxiety during early withdrawal from binge-drinking in adult and adolescent male mice. Drug Alcohol Depend. 184, 1-11. doi: 10. 1016/j.drugalcdep.2017.10.031

Lee, K. M., Coelho, M. A., Sern, K. R., Class, M. A., Bocz, M. D., and Szumlinski, K. K. (2017). Anxiolytic effects of buspirone and MTEP in the porsolt forced swim test. Chronic Stress. doi: 10.1177/2470547017712985 Epub ahead of print.

Lu, Y. M., Jia, Z., Janus, C., Henderson, J. T., Gerlai, R., Wojtowicz, J. M., et al. (1997). Mice lacking metabotropic glutamate receptor 5 show impaired learning and reduced CA1 long-term potentiation (LTP) but normal CA3 LTP. J. Neurosci. 17, 5196-5205. doi: 10.1523/JNEUROSCI.17-13-05196.1997

Manahan-Vaughan, D., and Braunewell, K.-H. (2005). The metabotropic glutamate receptor, mGluR5, is a key determinant of good and bad spatial learning performance and hippocampal synaptic plasticity. Cereb. Cortex 15, 1703-1713. doi: $10.1093 /$ cercor/bhi047

Matosin, N., Fernandez-Enright, F., Lum, J. S., and Newell, K. A. (2017). Shifting towards a model of mGluR5 dysregulation in schizophrenia: consequences for future schizophrenia treatment. Neuropharmacology 115, 73-91. doi: 10.1016/j. neuropharm.2015.08.003

Mesic, I., Guzman, Y. F., Guedea, A. L., Jovasevic, V., Corcoran, K. A., Leaderbrand, K., et al. (2015). Double dissociation of the roles of metabotropic glutamate receptor 5 and oxytocin receptor in discrete social behaviours. Neuropsychopharmacology 40, 2337-2346. doi: 10.1038/npp.2015.81

Meyer-Lindenberg, A., Mervis, C. B., Berman, K. F. (2006). Neural mechanisms in Williams syndrome: a unique window to genetic influences on cognition and behaviour. Nat. Rev. Neurosci. 7, 380-393. doi: 10.1038/nrn1906

Moy, S. S., Nadler, J. J., Perez, A., Barbaro, R. P., Johns, J. M., Magnuson, T. R., et al. (2004). Sociability and preference for social novelty in five inbred strains: an approach to assess autistic-like behaviour in mice. Genes Brain Behav. 3, 287-302. doi: 10.1111/j.1601-1848.2004. 00076.x
Nadler, J. J., Moy, S. S., Dold, G., Trang, D., Simmons, N., Perez, A., et al. (2004). Automated apparatus for quantitation of social approach behaviours in mice. Genes Brain Behav. 3, 303-314. doi: 10.1111/j.1601-183X.2004.00071.x

Nagel, J., Greco, S., Parsons, C. G., Flik, G., Tober, C., Klein, K. U., et al. (2015). Brain concentrations of mGluR5 negative allosteric modulator MTEP in relation to receptor occupancy - Comparison to MPEP. Pharmacol. Rep. 67, 624-630. doi: 10.1016/j.pharep.2015.01.004

Newman, M. E. J., and Girvan, M. (2004). Finding and evaluating community structure in networks. Phys. Rev. E Stat. Nonlin. Soft Matter Phys. 69:026113. doi: 10.1103/PhysRevE.69.026113

Nicoletti, F., Bockaert, J., Collingridge, G. L., Conn, P. J., Ferraguti, F., Schoepp, D. D., et al. (2011). Metabotropic glutamate receptors: from the workbench to the bedside. Neuropharmacology 60, 1017-1041. doi: 10.1016/j.neuropharm. 2010.10.022

Olsen, C. M., Childs, D. S., Stanwood, G. D., and Winder, D. G. (2010). Operant sensation seeking requires metabotropic glutamate receptor 5 (mGluR5). PLoS One 5:e15085. doi: 10.1371/journal.pone.0015085

Pollard, M., Bartolome, J. M., Conn, P. J., Steckler, T., and Shaban, H. (2014). Modulation of neuronal microcircuit activities within the medial prefrontal cortex by mGluR5 positive allosteric modulator. J. Psychopharmacol. 28, 935946. doi: $10.1177 / 0269881114542856$

Power, J. D., Schlaggar, B. L., Lessov-Schlaggar, C. N., and Petersen, S. E. (2013). Evidence for hubs in human functional brain networks. Neuron 79, 798-813. doi: 10.1016/j.neuron.2013.07.035

Rogers-Carter, M. M., Varela, J. A., Gribbons, K. B., Pierce, A. F., McGoey, M. T., Ritchey, M., et al. (2018). Insular cortex mediates approach and avoidance responses to social affective stimuli. Nat. Neurosci. 21, 404-414. doi: 10.1038/ s41593-018-0071-y

Silverman, J. L., Smith, D. G., Rizzo, S. J. S., Karras, M. N., Turner, S. M., Tolu, S. S., et al. (2012). Negative allosteric modulation of the mGluR5 receptor reduces repetitive behaviors and rescues social deficits in mouse models of autism. Sci. Transl. Med. 4:131ra51. doi: 10.1126/scitranslmed.300 3501

Slattery, D. A., Neumann, I. D., Flor, P. J., and Zoicas, I. (2017). Pharmacological modulation of metabotropic glutamate receptor subtype 5 and 7 impairs extinction of social fear in a time-point-dependent manner. Behav. Brain Res. 328, 57-61. doi: 10.1016/j.bbr.2017.04.010

Sreepathi, H. K., and Ferraguti, F. (2012). Subpopulations of neurokinin 1 receptor-expressing neurons in the rat lateral amygdala display a differential pattern of innervation from distinct glutamatergic afferents. Neuroscience 203, 59-77. doi: 10.1016/j.neuroscience.2011. 12.006

Stachowicz, K., Gołembiowska, K., Sowa, M., Nowak, G., Chojnacka-Wójcik, E., and Pilc, A. (2007). Anxiolytic-like action of MTEP expressed in the conflict drinking Vogel test in rats is serotonin dependent. Neuropharmacology 53, 741-748. doi: 10.1016/j.neuropharm.2007.08.002

Tanimizu, T., Kenney, J. W., Okano, E., Kadoma, K., Frankland, P. W., and Kida, S. (2017a). Functional connectivity of multiple brain regions required for the consolidation of social recognition memory. J. Neurosci. 37, 4103-4116. doi: 10.1523/JNEUROSCI.3451-16.2017

Tanimizu, T., Kono, K., and Kida, S. (2017b). Brain networks activated to form object recognition memory. Brain Res. Bull. 141, 27-34. doi: 10.1016/j. brainresbull.2017.05.017

Tao, J., Wu, H., Coronado, A. A., de Laittre, E., Osterweil, E. K., Zhang, Y., et al. (2016). Negative allosteric modulation of mGluR5 partially corrects pathophysiology in a mouse model of Rett syndrome. J. Neurosci. 36, 1194611958. doi: 10.1523/JNEUROSCI.0672-16.2016

Thomas, A. M., Bui, N., Graham, D., Perkins, J. R., Yuva-Paylor, L. A., and Paylor, R. (2011). Genetic reduction of group 1 metabotropic glutamate receptors alters select behaviors in a mouse model for fragile $\mathrm{X}$ syndrome. Behav. Brain Res. 223, 310-321. doi: 10.1016/j.bbr.2011.04.049

Tian, D., Stoppel, L. J., Heynen, A. J., Lindemann, L., Jaeschke, G., Mills, A. A., et al. (2015). Contribution of mGluR5 to pathophysiology in a mouse model of human chromosome 16p11.2 microdeletion. Nat. Neurosci. 18, 16-19. doi: $10.1038 / \mathrm{nn} .3911$

Vetere, G., Kenney, J. W., Tran, L. M., Xia, F., Steadman, P. E., Parkinson, J., et al. (2017). Chemogenetic interrogation of a brain-wide fear memory 
network in mice. Neuron 94, 363-374.e4. doi: 10.1016/j.neuron.2017. 03.037

Vicidomini, C., Ponzoni, L., Lim, D., Schmeisser, M. J., Reim, D., Morello, N., et al. (2017). Pharmacological enhancement of mGlu5 receptors rescues behavioral deficits in SHANK3 knock-out mice. Mol. Psychiatry 22, 689-702. doi: 10.1038/ mp.2016.30

Youssef, E. A., Berry-Kravis, E., Czech, C., Hagerman, R. J., Hessl, D., Wong, C. Y., et al. (2018). Effect of the mGluR5-NAM basimglurant on behavior in adolescents and adults with fragile $\mathrm{X}$ syndrome in a randomized, double-blind, placebo-controlled trial: FragXis phase 2 results. Neuropsychopharmacology 43, 503-512. doi: 10.1038/npp.2017.177
Conflict of Interest Statement: The authors declare that the research was conducted in the absence of any commercial or financial relationships that could be construed as a potential conflict of interest.

Copyright ( 2019 Ramos-Prats, Kölldorfer, Paolo, Zeidler, Schmid and Ferraguti. This is an open-access article distributed under the terms of the Creative Commons Attribution License (CC BY). The use, distribution or reproduction in other forums is permitted, provided the original author(s) and the copyright owner(s) are credited and that the original publication in this journal is cited, in accordance with accepted academic practice. No use, distribution or reproduction is permitted which does not comply with these terms. 